\title{
Role of immunohistochemistry and apoptosis as investigative tools in assessing the prognosis of patients with prostate tumours
}

\author{
SRIKUMAR CHAKRAVARTHI ${ }^{1}$, P.M. THANI ${ }^{1}$, DAVID LOW WEE YANG ${ }^{2}$, \\ LINDA TJOA HUSIN ${ }^{2}$ and NAGARAJA LEE ${ }^{3}$
}

\author{
Departments of ${ }^{1}$ Pathology, ${ }^{2}$ Postgraduate Studies, Research Laboratory, and ${ }^{3}$ Community Medicine, \\ Faculty of Medicine, International Medical University, Bukit Jalil, 57000 Kuala Lumpur, Malaysia
}

Received November 27, 2009; Accepted December 23, 2009

DOI: 10.3892/etm_00000061

\begin{abstract}
Apoptosis is a form of programmed cell death necessary for the regulation of the size of organs in adult life. Disruption of apoptotic pathways has been suggested as an important regulatory mechanism in prostatic tumours. The aim of this study was to examine the expression of apoptosisregulating genes bcl-2 and p53 using immunohistochemistry, and the Gleason score in core needle biopsy specimens of prostate adenocarcinoma. We studied bcl-2 and p53 expression in 30 cases of low-, 30 cases of intermediate- and 20 cases of high-grade prostate adenocarcinoma. Overexpression of bcl-2 and p53 were noted in 54 and 61 of 80 patients (67.5 and $76.25 \%$ ), respectively. The statistical analysis of the present data suggested that there is significant relation between p53 and bcl-2 expression, and Gleason score in prostate cancer. Thus, immunohistochemistry is a useful investigative parameter in assessing apoptosis to analyse the prognosis of prostatic tumours.
\end{abstract}

\section{Introduction}

Prostate adenocarcinoma is a common malignant disease particularly in industrialized countries. Rates of detection of prostate cancer vary widely worldwide, with detection less frequent in South and East Asia compared to Europe, and in particular compared to the United States. Prostate cancer tends to develop in men over the age of 50 years, and is one of the most prevalent types of cancer among men. Prostate cancer patients may never have symptoms, and as a result undergo no therapy, eventually succumbing to the disease (1).

The biological behavior of prostate cancer is unpredictable in individual patients, ranging from slow growing non-life-

Correspondence to: Dr Srikumar Chakravarthi, Department of Pathology, Faculty of Medicine, International Medical University, Bukit Jalil, 57000 Kuala Lumpur, Malaysia

E-mail: srikumar_chakravarthi@imu.edu.my

Key words: prostate, adenocarcinoma, apoptosis, immunohistochemistry threatening to highly aggressive cancers (2). At present, the most established prognostic factors for prostate cancer include histological grade (Gleason system) and tumour stage (3).

Cellular proliferation and programmed cell death (apoptosis) are associated with tumour growth in general, and with prostate cancer growth in particular. It has been demonstrated that protein expression of the proto-oncogene bcl-2 (a potent inhibitor protein against apoptosis) and of the tumour-suppressor gene p53 (a regulator of cellular proliferation and apoptosis) are useful prognostic indicators of the progression of prostate cancer $(4,5)$.

Although studies have strongly suggested that molecular analyses can provide useful prognostic information when large harvested biopsy samples or the entire prostate are examined, there is little information regarding the clinical significance of these molecular examinations in core needle biopsies. This approach is potentially impeded by tumour heterogeneity, since small tumour fragments may not be representative of the entire carcinoma. However, molecular analyses should be performed prior to undertaking an aggressive surgical approach, as treatment options may be influenced by the molecular analyses. Prognostic factors would be particularly useful if they could be evaluated from core needle biopsy specimens supported by transrectal ultrasonography (6).

The goal of the present study was to evaluate the role of immunohistochemistry as an effective investigative marker for the study of the relationship between expression of apoptosis- regulating genes bcl-2 and p53 and Gleason score in prostate cancer.

\section{Materials and methods}

Patients. Core needle biopsy specimens of 80 previously untreated prostate cancer patients acquired from the archives of the Department of Pathology of our teaching hospital were retrospectively assessed (Table I) after obtaining informed consent from the patients, and prior approval and necessary clearance from the research and ethical committees of the university. Digital rectal examination, serum PSA (ng/ml), transrectal ultrasonography, whole body bone scaning and pelvic computerized tomography (optional) were used for the clinical staging of the disease. 
Table I. Gleason score and the number of patients in each category.

\begin{tabular}{lc}
\hline Gleason score & Patients \\
\hline $2-4$ (Gleason grade I) & 30 \\
$5-7$ (Gleason grade II) & 30 \\
$8-10$ (Gleason grade III) & 20 \\
\hline
\end{tabular}

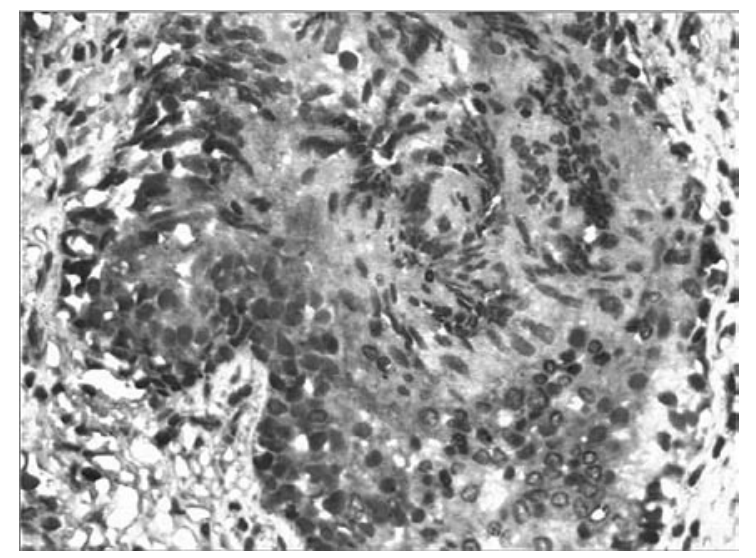

Figure 1. Photomicrograph showing a large tumour gland exhibiting cytoplasmic positivity for bcl-2 (immunohistochemistry, $\mathrm{x} 400$ ).

Histopathology and immunohistochemistry; Gleason score. Core needle prostatic biopsy specimens prior to initiation of any treatment were obtained for this study. All slides were reviewed, and a Gleason score was determined by adding the numbers for the two most predominant patterns. Immunohistochemical stains were performed on $5-\mu \mathrm{m}$ sections of the formalin-fixed, paraffin-embedded biopsy specimens. Sections on silanized glass slides were deparaffinized in xylene and rehydrated using ethanol gradients. The sections were pretreated with antigen target retrieval solution at $90^{\circ} \mathrm{C}$ for $40 \mathrm{~min}$ in citrate buffer $(10 \mathrm{mmol} / \mathrm{l}$ citric acid monohydrate adjusted with $2 \mathrm{~N}$ sodium hydroxide to $\mathrm{pH}$ 6.0). Endogenous peroxidase activity was blocked by methanol with $0.3 \%$ hydrogen peroxide for $30 \mathrm{~min}$. The slides were incubated for 45 min with the primary antibodies: mouse monoclonal antibcl-2 antibody (clone 124; Dako) and anti-p53 antibody (clone DO-7; Dako). The anti-p53 antibody DO-7 recognizes an epitope on the $\mathrm{N}$-terminus of the $\mathrm{p} 53$ protein and reacts with wild-type and mutant p53 proteins. The anti-bcl-2 antibody 124 reacts specifically with bcl-2 oncoprotein. The working dilution of these antibodies was 1:50 and 1:40, respectively. The slides were then incubated with a biotinylated rabbit antimouse immunoglobin for $30 \mathrm{~min}$ at room temperature. They were subsequently incubated using the streptoavidine-biotin method. The sections were counterstained with hematoxylin.

p53-positive colon adenocarcinoma served as a positive control for p53 immunostaining, and prostatic basal cells as an internal control for bcl-2 immunostaining. All slides were evaluated by two pathologists. Specimens were considered to be bcl-2-positive when $>10 \%$ of the tumour cells stained for bcl-2. p53-positivity was established when $>10 \%$ of prostate tumour cells demonstrated nuclear reactivity $(7,8)$.

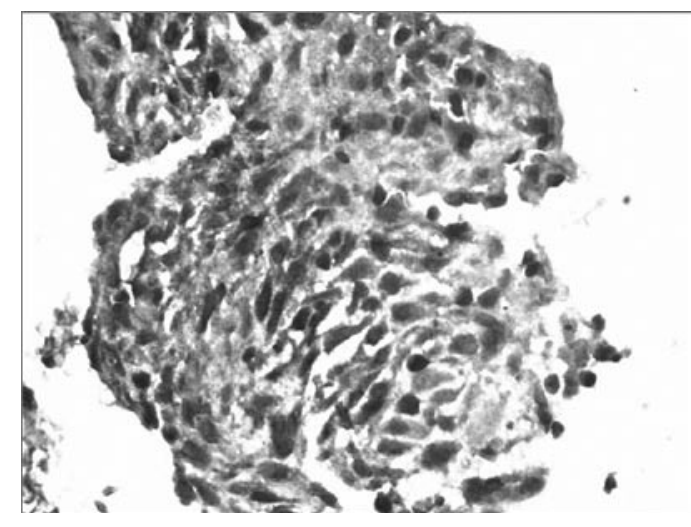

Figure 2. Photomicrograph showing a large cluster of tumour cells showing nuclear positivity for $\mathrm{p} 53$ (immunohistochemistry, $\mathrm{x} 400$ ).

Statistical analysis. Statistics were performed using the Fisher's $\chi^{2}$ test. The patient population was divided into several groups according to bcl-2 immunostaining, p53 immunostaining and Gleason score.

\section{Results}

The mean age and serum PSA values of 80 patients, whose samples were retrospectively obtained from clinical archives, were $72.3 \pm 8.2$ years (range $54-83$ years) and $36.5 \pm 18.3 \mathrm{ng} / \mathrm{ml}$ (5.94-120.00 ng/ml), respectively. Overexpression of bcl-2 was determined in 54 of the 80 cases $(67.5 \%$ ), of which 18 were in grade III, 16 in grade II and 20 in grade I. Expression of p53 was noted in 61 of the 80 cases $(76.25 \%)$, of which 18 were in grade III, 18 in grade II and 25 in grade I. The relationship between increased levels of apoptosis-regulator proteins (p53 and bcl-2) and Gleason score was statistically significant $(\mathrm{p}<0.0014)$.

\section{Discussion}

While pathological stage, grade, positive surgical margins and tumour volume are perhaps the most commonly accepted prognostic factors after radical prostatectomy, they cannot be used preoperatively. An approach to developing immunohistochemistry for prognostic markers correlating with biological aggressiveness is greatly needed. Among the several potential targets, regulators of the apoptotic pathway, including bcl-2 and p53, have been at the forefront of prostate cancer research, and have recently been evaluated as prognostic markers. p53 and bcl-2 overexpression has been investigated independently in a large number of different malignancies for its potential value as a prognostic marker.

In high-grade prostatic adenocarcinomas, studies have suggested that combined expression of bcl-2 and p53 protein is associated with poor patient prognosis, more so than p53 accumulation alone $(6,9,10)$. In an investigation of the extent of apoptosis in a set of testicular and ovarian germ cell tumours compared to the expression of p53 and bcl-2, the results revealed that the extent of apoptosis was highest in embryonal carcinomas followed by seminomas, choriocarcinomas and immature teratomas (11). Embryonal carcinomas also showed quantitatively the strongest p53 expression. bcl-2 was only expressed in teratomas and might partly counteract apoptosis in these tumours. 
There have been several studies of the prognostic significance of bcl-2 and p53 in prostate cancer. Nearly all of these studies evaluated only p53-positivity in needle biopsy, transurethral prostate resection or radical prostatectomy specimens in order to draw prognostic conclusions. In the present study, we analysed only pretreatment prostate needle biopsies. Another study examined the expression of p53 in 109 prostate cancers and concluded that mutation of p53 may be involved in the development of certain prostate cancers. It was found that patients whose prostate cancers showed p53 immunoreactivity had a significantly worse prognosis than patients with p53-negative cancers (12).

A study of 18 patients with locally recurrent prostate carcinoma after radiotherapy found that $72 \%$ had p53 nuclear immunoreactivity, while all 5 patients with available pre-RT biopsies had p53 immunoreactivity (13). Cheng et al examined p53 abnormalities using immunohistochemistry in lymph node-positive prostate cancer. They found that a significant proportion of primary tumours (52\%) and matched lymph node metastases $(58 \%)$ showed nuclear accumulation of the p53 protein (14).

However, other studies examining the expression of p53 in 55 prostate cancers concluded that it was not a useful prognostic indicator, and also found no association between p53 expression and patient outcome (15-17). By contrast, in a study conducted by Bauer et al (17) examining p53 and bcl-2 immunoreactivity in 175 radical prostatectomy specimens, aberrant bcl-2 was observed in $27 \%$ of the cancer cases. Within 5 years, $67 \%$ of these patients had a relapse, while only $31 \%$ of those with bcl-2-negative cancers relapsed. In another study, aberrant p53 expression was observed in $65 \%$ of cancer cases, of which $51 \%$ had a relapse, while $78 \%$ of patients with p53-negative cancers did not have disease progression. When expression rates for $\mathrm{p} 53$ and bcl-2 were combined, the 5 -year failure rate was $75.3 \%$. The authors found a statistically significant difference between p53 and bcl-2-positive and -negative patients. They reported that overexpression of bcl-2 was not directly associated with p53 protein accumulation in adenocarcinoma of the prostate (18).

There have also been studies on the Gleason score and apoptosis-regulator proteins in prostate cancer. In a study of p53 and bcl-2 immunoreactivity in 146 prostatic carcinomas, bcl-2- and p53-positivity was found in 20 and 27\% of the 146 prostate cancers, respectively. Both bcl-2- and p53-positivity were found only in 5\% of the cases. The researchers reported not only that p53-positivity was associated with advancing Gleason grade, but also that bcl-2-positivity was found exclusively in moderately to poorly differentiated (Gleason score 6-10) tumours. However, there was no statistically significant correlation between bcl-2-positivity and Gleason score $(18,19)$.

Our study found a highly significant correlation between the expression of the apoptosis-regulating genes bc1-2 and p53 and Gleason score in adenocarcinomas of the prostate. Further confirmation of the significance of these molecular examinations in more homogeneous and larger patient groups is required.

\section{Acknowledgements}

This study was partly conducted with the aid of a research grant (BMS I-01-2007/07-08) provided by the International Medical University, Kuala Lumpur, Malaysia. The authors also wish to acknowledge the contribution of Dr J. Karthikesh, JRD Pathology Laboratory, for his expert advise.

\section{References}

1. Miller DC, Hafez KS and Stewart A: Prostate carcinoma presentation, diagnosis and staging: an update from the National Cancer Data Base. Cancer 98: 1169-1172, 2003.

2. Von Eschenbach AC: The biologic dilemma of early carcinoma of prostate. Cancer 78: 326-329, 1996.

3. Grignon DJ and Hammond EH: College of American Pathologists Conference XXVI on clinical relevance of prognostic markers in solid tumors. Report of the Prostate Cancer Working Group. Arch Pathol Lab Med 119: 1122-1126, 1995.

4. Hockenbery D, Nunez C and Milliam C: Bcl-2 is an inner mitochondrial membrane protein that blocks programmed cell death. Nature 348: 334-338, 1990.

5. LevineAJ: p53, the cellular gatekeeper for growth and division. Cell 88: 323-325, 1997.

6. Issacs JT: Molecular markers for prostate cancer metastasis: developing diagnostic methods for predicting the aggressiveness of prostate cancer. Am J Pathol 150: 1511-1521, 1997.

7. Grossfeld CD, Olumi AF and Connolly JA: Locally recurrent prostate tumours following either radiation therapy or radical prostatectomy have changes in Ki-67 labelling index, p53 and bcl-2 immunoreactivity. J Urol 159: 1437-1443, 1998.

8. Chakravarthi S, Linda TH, Thani PM and Nadeem IB: Role of apoptosis and abnormalities in expression of bcl-2 in benign nodular hyperplasia of prostate. Res J of Biol Sci 4: 836-841, 2009.

9. Piris MA, Pezzella F and Martinez Montero JC: p53 and bcl2 expression in high grade B-cell lymphomas. Br J Cancer 69: 337, 1994.

10. Masuda M, TakanoY and Iki M: Prognostic significance of Ki-67, p53 and bcl-2 expression in prostate cancer patients with lymph node metastases: a retrospective immunohistochemical analysis. Pathol Int 48: 41-46, 1998.

11. Soini Y and Paakko P: Extent of apoptosis in relation to p53 and bcl-2 expression in germ cell tumors. Hum Pathol 27: 1221-1226, 1996.

12. Shurbaji MS, Kalbfeisch JH and Thurmond S: Immunohistochemical detection of p53 protein as a prognostic indicator in prostate cancer. Hum Pathol 26: 106-109, 1999.

13. Prendergest NJ, Atkins MR and Schatte EC: p53 immunohistochemical and genetic alterations are associated at high incidence with post-irradiated locally persistent prostate carcinoma. J Urol 155: 1685-1692, 1996.

14. Cheng L, Sebo JT and Pisanlsky T: p53 protein overexpression is associated with increased cell proliferation in patients with locally recurrent prostate carcinoma after radiation therapy. Cancer 85: 1293-1299, 1999.

15. Fox SB, Persad RA and Royds J: p53 and c-myc expression in stage Al prostatic adenocarcinoma: useful prognostic determinants? J Urol 150: 490-494, 1993.

16. Kallakury BV, Figge J and Ross JS: Association of p53 immunoreactivity with high Gleason tumor grade in prostatic adenocarcinoma. Hum Pathol 25: 92-96, 1994.

17. Bauer JJ, Sesterhenn IA and Mostofi FK: Elevated levels of apoptosis regulator proteins p53 and bcl-2 are independent prognostic biomarkers in surgically treated clinically localized prostate cancer. J Urol 156: 1511-1516, 1996.

18. Matsushima N, Kitamura $\mathrm{T}$ and Goto $\mathrm{T}$ : Combined analysis with bcl-2 and p53 immunostaining predicts poorer prognosis in prostatic carcinoma. J Urol 158: 2278-2283, 1997.

19. Bubendorf L, Sauter G and Moch H: Prognostic significance of bcl-2 in clinically localized prostate cancer. Am J Pathol 148: $1557,1996$. 